\title{
Pupil-occluding organized hyphema anterior to the intraocular lens treated by neodymium: yttrium-aluminum-garnet laser: a case report
}

This article was published in the following Dove Press journal:

International Medical Case Reports Journal

7 July 2016

Number of times this article has been viewed

\author{
Moosang Kim' \\ Seung-Jun Lee' \\ Sang Beom Han' \\ Hee Kyung Yang ${ }^{2}$ \\ Joon Young Hyon ${ }^{2}$ \\ 'Department of Ophthalmology, \\ Kangwon National University \\ Hospital, Kangwon National \\ University Graduate School of \\ Medicine, Chuncheon, ${ }^{2}$ Department \\ of Ophthalmology, Seoul National \\ University Bundang Hospital, Seoul \\ National University College of \\ Medicine, Seongnam, South Korea
}

Correspondence: Sang Beom Han Department of Ophthalmology, Kangwon National University Hospital, 156 Baengnyeong-ro, Chuncheon, Kangwon 200-722, South Korea

$\mathrm{Tel}+82332589210$

Fax +82 332582296

Email m.sangbeom.han@gmail.com

\begin{abstract}
We report, to our best knowledge, the first case of treatment of pupil-occluding postoperative organized hyphema anterior to the intraocular lens (IOL) using neodymium: yttrium-aluminum-garnet (Nd:YAG) laser. A 78-year-old Asian female underwent uneventful cataract operation. She had been taking aspirin, which she discontinued 1 week before surgery. Iris prolapse occurred at the end of the surgery, which led to intracameral bleeding. Two weeks later, her best-corrected visual acuity was hand motion. Although hyphema had decreased, pupiloccluding organized hematoma had formed anterior to the IOL. The blood clot anterior to the IOL was removed using Nd:YAG laser. One week later, although the hematoma anterior to the IOL resolved, endocapsular hematoma was observed, which was dispersed with Nd:YAG laser posterior capsulotomy. Two weeks later, her best-corrected visual acuity improved to 20/60. There was no complication associated with $\mathrm{Nd}$ :YAG laser. In conclusion, pupil-occluding organized hyphema anterior to the IOL can occur as a complication of cataract surgery, in which Nd:YAG laser can be a useful treatment option.
\end{abstract}

Keywords: blood clot, case report, hyphema, Nd:YAG laser, postoperative hyphema

\section{Introduction}

In ophthalmologic practice, neodymium: yttrium-aluminum-garnet ( $\mathrm{Nd}: \mathrm{YAG}$ ) laser is mainly applied for treatment of posterior capsular opacity. ${ }^{1}$ It has also been used for a variety of indications, including peripheral iridotomy, ${ }^{2}$ anterior vitreolysis, ${ }^{3}$ anterior capsule relaxing incisions, ${ }^{4}$ anterior capsulotomy in white intumescent cataracts, ${ }^{5}$ goniopuncture, ${ }^{6}$ removal of residual cortex, ${ }^{7}$ and treatment of endocapsular hematoma. ${ }^{8}$

However, to our best knowledge, the treatment of organized hyphema anterior to intraocular lens (IOL) using Nd:YAG laser has never been reported. Pupil-occluding organized hyphema anterior to the IOL conceivably causes serious vision decrease. However, surgical intervention can often be difficult due to the risk of rebleeding, and other treatment options should be considered. Herein we present a case of successful treatment of pupil-occluding organized hyphema anterior to the IOL by direct application of $\mathrm{Nd}$ :YAG laser to the hematoma.

\section{Case presentation}

A 78-year-old Asian female underwent cataract surgery in the right eye (oculus dexter [OD]). Preoperatively, her best-corrected visual acuity (BCVA) was 20/400 OD. She had a history of ischemic heart disease and hypertension and, therefore, was taking aspirin. She discontinued aspirin 1 week preoperatively, and coagulation panels 
were within normal range. Cataract surgery was performed with topical anesthesia and clear corneal incision, and was uneventful until IOL implantation and removal of viscoelastics. However, she had intraoperative floppy iris syndrome and iris prolapse occurred through the main wound at the conclusion of the surgery, which led to intracameral hemorrhage. Although copious irrigation of the anterior chamber combined with aspiration of the blood clot was done, bleeding was not completely controlled, and eventually, hyphema filled half of the intracameral space. Postoperatively, oral prednisolone (30 $\mathrm{mg}$ a day for 3 days) and topical prednisolone acetate ( $1 \%$, six times a day) were prescribed.

One week later, her BCVA was hand motion, and intraocular pressure (IOP) measured with Goldmann applanation tonometry (GAT) was $15 \mathrm{mmHg}$. Although hyphema had decreased, organized hyphema anterior to the IOL had occluded the pupil (Figure 1A). One week later, her BCVA was hand motion, and there was no change in the organized hyphema anterior to the IOL. Using Nd:YAG laser (3.0-4.0 mJ), the organized hemorrhage on the IOL was lysed. One week later, her BCVA was 20/400, and IOP was $15 \mathrm{mmHg}$ by GAT. Hematoma anterior to the IOL was completely absorbed. However, endocapsular hematoma that occluded two-thirds of the pupil was observed (Figure 1B). Nd:YAG laser posterior capsulotomy $(1.0-2.0 \mathrm{~mJ})$ was performed to disperse the blood clot from the visual axis. Two weeks later, her BCVA improved to 30/60, and IOP was 14 mmHg by GAT. There was no blood occluding the visual axis, and hyphema was completely absorbed (Figure 1C). There was no hemorrhage in the vitreous cavity. No evidence of corneal endothelial decompensation was observed.

Written informed consent was obtained from the patient for publication of this case report and any accompanying images. As this is a single case report and only retrospective review of medical records was needed, ethics approval was waived by the institutional review board of Kangwon National University Hospital.

\section{Discussion}

Hyphema following cataract surgery is uncommon. Studies showed that phacoemulsification of uncomplicated cataract with IOL implantation with topical anesthesia and clear corneal incision can be safe even in patients taking anticoagulants or antiplatelet drugs. ${ }^{9}$ However, in conditions that may increase the risk of bleeding, such as small pupil, floppy iris syndrome, iris neovascularization, or pseudoexfoliation or interruption of antiplatelet or anticoagulant therapy, the risk of postoperative hematoma should be considered. ${ }^{9}$ Although aspirin was stopped and coagulation panels were normal in our patient, inadvertent intracameral hemorrhage occurred associated with iris prolapse. Complete removal of the hemorrhage was impossible, which led to blood collection in the anterior chamber and capsular bag. Although the hemorrhage was partly absorbed, remnant organized hyphema anterior to the IOL occluded the pupil and led to substantial decrease in vision. Endocapsular hematoma also caused occlusion of the visual axis. Secondary operation to wash out the hemorrhage could be a treatment option. However, considering that the patient had a risk for iris prolapse, additional surgical manipulation could exacerbate the hyphema. Conservative management until the hemorrhage is completely absorbed could be another option. However, the organized hyphema anterior to the IOL persisted for 2 weeks, and reduced her vision significantly by occluding the pupil. There was also a possibility that the organized hemorrhage anterior to the IOL had formed a persistent fibrin membrane, necessitating intervention to improve the condition. Eventually, the remaining organized hemorrhage anterior and posterior to the IOL was removed using Nd:YAG laser. We chose lysis of the hematoma anterior to the IOL by direct application of Nd:YAG laser because it can deliver strong energy to a small target without the risk of damage to the adjacent tissue.

As in the present case, intraoperative hyphema cannot be completely prevented, and in these circumstances, secondary

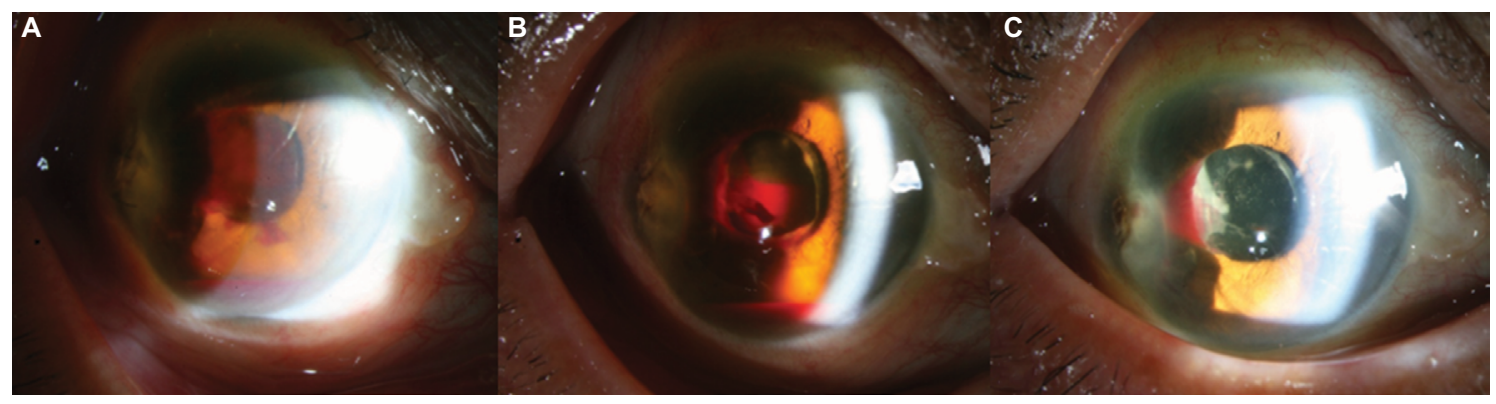

Figure I Change of the hyphema before and after the laser treatment.

Notes: (A) Anterior segment photography taken I week postoperatively, demonstrating organized hyphema anterior to the IOL occluding pupil. (B) Anterior segment photography taken at I week after the lysis of organized hyphema anterior to the IOL. Although hematoma anterior to the IOL was absorbed, endocapsular hemorrhage remained. (C) Anterior segment photography taken at 2 weeks after the posterior capsulotomy. Hematoma anterior and posterior to the IOL completely resolved. Abbreviation: IOL, intraocular lens. 
operation may often be difficult due to the risk of rebleeding. Surgical intervention of intracameral hemorrhage can sometimes be dangerous also in patients with increased bleeding tendency, such as hemophilia or liver diseases. In these patients, $\mathrm{Nd}$ :YAG laser can be a safe option for vision recovery.

$\mathrm{Nd}$ :YAG laser was also used for the treatment of postoperative endocapsular hematoma. ${ }^{8}$ However, in these cases, the laser was used to make posterior capsular opening and to drain the blood to the vitreous cavity, as we did for the treatment of endocapsular hematoma. ${ }^{8}$ For the treatment of organized hyphema anterior to the IOL, we directly applied the Nd:YAG laser for the lysis of the hematoma, and thus used higher energy compared to the energy level used for posterior capsulotomy.

Although Nd:YAG laser posterior capsulotomy is relatively safe, complications including retinal detachment, macular edema, optic disc edema, uveitis, glaucoma, macular hole, and endophthalmitis have been reported..$^{10}$ In the present case, the energy of the laser was concentrated on the blood clot and the possibility of damage to other tissues was low. However, as relatively stronger energy was used, we believe attention should be paid to the possibility of complications.

\section{Conclusion}

In conclusion, pupil-occluding organized hyphema anterior to the IOL can occur as a complication of cataract surgery, although it may be rare. Direct lysis of the hematoma using Nd:YAG laser can be a useful treatment option in this condition, particularly when surgical intervention is difficult due to the risk of rebleeding.

\section{Author contributions}

MK carried out data acquisition and drafted the manuscript. SJL interpreted the data and drafted the manuscript.
SBH interpreted the data and drafted the manuscript. HKY analyzed the data and critically revised the manuscript. JYH analyzed the data and critically revised the manuscript. All authors read and approved the final manuscript, and agree to be accountable for all aspects of the work in ensuring that questions related to the accuracy or integrity of any part of the work are appropriately investigated and resolved.

\section{Disclosure}

The author reports no conflicts of interest in this work.

\section{References}

1. Aslam TM, Devlin H, Dhillon B. Use of Nd:YAG laser capsulotomy. Surv Ophthalmol. 2003;48:594-612.

2. Gilbert CM, Robin AL, Pollack IP. Iridotomy using the Q-switched neodymium (Nd):YAG laser. Ophthalmology. 1984;91:1123.

3. Steinert RF, Wasson PJ. Neodymium:YAG laser anterior vitreolysis for Irvine-Gass cystoid macular edema. J Cataract Refract Surg. 1989;15:304-307.

4. Hayashi K, Yoshida M, Nakao F, Hayashi H. Prevention of anterior capsule contraction by anterior capsule relaxing incisions with neodymium:yttrium-aluminum-garnet laser. Am JOphthalmol. 2008;146: 23-30.

5. Coelho RP, Martin LF, Paula JS, Scott IU. Comparison of preoperative Nd:YAG laser anterior capsulotomy versus two-stage curvilinear capsulorhexis in phacoemulsification of white intumescent cataracts Ophthalmic Surg Lasers Imaging. 2009;40:582-585.

6. Anand N, Pilling R. Nd:YAG laser goniopuncture after deep sclerectomy: outcomes. Acta Ophthalmol. 2010;88:110-115.

7. Hood CT, Shtein RM, Mian SI, Sugar A. Neodymium-yttrium-aluminum-garnet laser lysis of retained cortex after phacoemulsification cataract surgery. Am J Ophthalmol. 2012;154:808-813 e1.

8. Hagan JC, 3rd, Gaasterland DE. Endocapsular hematoma. Description and treatment of a unique form of postoperative hemorrhage. Arch Ophthalmol. 1991;109:514-518.

9. Grzybowski A, Ascaso FJ, Kupidura-Majewski K, Packer M. Continuation of anticoagulant and antiplatelet therapy during phacoemulsification cataract surgery. Curr Opin Ophthalmol. 2015;26:28-33.

10. Han SB, Yang HK, Hyon JY, Woo SJ, Wee WR. Transient optic disc edema following neodymium: yttrium-aluminum-garnet laser posterior capsulotomy. Can J Ophthalmol. 2012;47:e1-2.
The International Medical Case Reports Journal is an international, peer-reviewed open-access journal publishing original case reports from all medical specialties. Previously unpublished medical posters are also accepted relating to any area of clinical or preclinical science. Submissions should not normally exceed 2,000 words or
4 published pages including figures, diagrams and references. The manuscript management system is completely online and includes a very quick and fair peer-review system, which is all easy to use. Visit $\mathrm{http}: / / \mathrm{www}$. dovepress.com/testimonials.php to read real quotes from published authors. 